\title{
Utility of 3D rotational angiography road map in flow diverter deployment in a distal dissecting MCA aneurysm
}

\author{
Oriela Rustemi, MD, ${ }^{1}$ Loris Di Clemente, MD, ${ }^{2}$ Fabio Raneri, MD, ${ }^{1}$ Lorenzo Volpin, MD, ${ }^{1}$ \\ and Giuseppe lannucci, MD²
}

Departments of ${ }^{1}$ Neurosurgery and ${ }^{2}$ Neuroradiology, San Bortolo Hospital, Vicenza, Italy

\begin{abstract}
Distal, dissecting, middle cerebral artery (MCA) aneurysms are changing surgically and endovascularly. Endovascular treatment requires flow diverter stenting. A good vessel visualization is crucial for safe navigation. Three-dimensional rotational digital subtraction angiography (3D-DSA) is used routinely in diagnostic imaging. The utilization of the 3DDSA road map in vessel navigation and stent deployment is novel. An illustrative video of a distal, dissecting left MCA aneurysm treated with flow diverter stenting is presented. The technical issues were distal location, dissecting nature with double lumen, proximal stenosis, and vessel curves. The 3D-DSA road map helped to enhance visualization with a safer procedure.
\end{abstract}

The video can be found here: https://youtu.be/sS301Z0P8WE.

KEYWORDS angiography; cerebral aneurysm; dissecting aneurysm; endovascular; flow diverter stent; video 